\title{
Terminology and notation conventions
}

I will use the term variety most of the time as a cover term for language, dialect (or lect of any sort), etc. When it is important for me to differentiate two languages, I will do that as best I can, but there is an unsolvable issue when it comes to more specific terms. Where such entities begin and end, and how to define them, is problematic, so I ask the reader's patience with my vagueness when I judge the issue to be irrelevant to whatever the current point is. I will generally use names for varieties that other authors use, although this can be contentiousas well; again I ask for patience in this matter. Most abbreviations are introduced in the text, and refer to variables, varieties, or key pieces of literature; below are some important notations used throughout.

[x] phonetically represented segment (see the International Phonetic Alphabet chart in Figure 0.1); this notation also represents variants of a variable

/x/ phonemically represented segment

$\{\mathrm{x}\}$ morpheme; $\{-\mathrm{x}\}$ represents a suffix and $\{\mathrm{x}-\}$ a prefix

(x) sociolinguistic variable 